Pueva Ptinexaxio Revista Digital de OFilosofia ISSN 1850-3578 2013 - Vol. 8 - Número VIII - Resistencia, Chaco, Argentina. Pp. 136 - 147

\title{
La filosofía de la historia kantiana como condición de imposibilidad de una ontología histórica de nosotros mismos
}

Guillermo A. Vega

Universidad Nacional del Nordeste (Argentina)

Recibido: 19/07/2013

Aceptado: $28 / 08 / 2013$

\section{Resumen}

El presente artículo recupera una serie de ejercicios de lectura que el filósofo francés Michel Foucault hiciera alrededor del texto kantiano Was ist Aufklärung? entre fines de la década de 1970 y comienzos de 1980. El objetivo principal de la operación foucaultiana consistía en indicar el punto de emergencia de una modalidad de pregunta filosófica que fue opacada por el problema más extendido acerca de las condiciones del conocimiento verdadero. El proyecto de una "ontología histórica de nosotros mismos" foucaultiano abreva, entonces, en la actitud que presupone la inquisición kantiana sobre las características del presente desde el cual se ejercita el pensamiento. Sin embargo, la lectura de Foucault deja de lado una serie de elementos teóricos que forman el trasfondo problemático sobre el cual se presenta el breve texto sobre la Ilustración. La tesis aquí defendida sostiene que en caso de actualizar el universo de problemas que circunscriben la pregunta por la llustración, el proyecto de una ontología histórica de nuestro presente, en términos foucaultianos, se volvería imposible.

\section{Abstract}

This paper revisit a series of reading exercises that french philosopher Michel Foucault -made between ends of the 1970s and beginning of 1980- regarding Kant's Was ist Aufklärung? The main objective of Foucault's procedure consisted in highlighting the emerging point of a philosophical questioning which was overshadowed by the most common problem of the conditions of true knowledge. Thus, Foucault's project of an "historical ontology of our own selves" derives from the attitude presupposed in the Kantian inquiry about the characteristics of the present from which thought is applied. Nonetheless, Foucault's reading put aside a series of theoretic elements that comprise a problematical background upon which the brief work on Enlightenment is exposed. The thesis here sustained says that in case of actualizing 
QNueva Etinexaxia Revista Digital de OFilosofia ISSN 1850-3578 2013 - Vol. 8 - Número VIII - Resistencia, Chaco, Argentina. Pp. 136 - 147

the universe of problems that circumscribe the question about Enlightenment, the project of an historical ontology of our present -in the terms of Foucault- would become impossible.

Pero dentro mismo de la filosofía moderna y contemporánea hay otro tipo de cuestión, otro modo de interrogación crítica: la que vemos surgir, justamente, en la cuestión de la Aufklärung o el texto de la revolución. Esta otra dirección crítica no plantea la cuestión de las condiciones 
Q Nueva Etinexaxio Revista Digital de OFilosofia ISSN 1850-3578 2013 - Vol. 8 - Número VIII - Resistencia, Chaco, Argentina. Pp. 136 - 147

en que es posible un conocimiento verdadero; es una tradición que pregunta: ¿qué es la actualidad? ¿Cuál es el campo actual de nuestras experiencias? ¿Cuál es el campo actual de las experiencias posibles? No se trata de una analítica de la verdad, se trataría de lo que podríamos llamar una ontología del presente, una ontología de la actualidad, una ontología de la modernidad, una ontología de nosotros mismos. ${ }^{1}$

Para M. Foucault, la pregunta kantiana sobre el significado de la llustración representa, si atendemos a las condiciones de su formulación, pararse en la vertical de su tiempo para reflexionar sobre lo que caracteriza al mismo. Este gesto kantiano se convierte en el momento inaugural de una nueva manera de problematizar las cosas en filosofía. La pregunta que indaga por el presente rastrea una especificidad, una nota característica, que pueda dar cuenta del momento actual frente al pasado y a un hipotético futuro. De aquí Foucault extrae los lineamientos de un programa filosófico que, anclado en los asuntos del presente, tratará de establecer las condiciones históricas de su configuración para ensayar modificaciones posibles.

La respuesta que da Kant a la pregunta “¿qué es la Ilustración?" interesa a Foucault en la medida en que puede decantar de la misma lo que denomina el "ethos filosófico de la modernidad", es decir, una actitud con respecto a la verdad consistente en "atreverse" a formularla, a producirla, en lugar de recibirla pasivamente. Dando un paso más en su peculiar lectura del texto kantiano, Foucault termina enlazando el sentido de la pregunta sobre la llustración con la respuesta que interpreta que Kant da de la misma; en otras palabras, la pregunta por la llustración no es más que la expresión del desarrollo de la respuesta kantiana a la luz de la operación de lectura foucaultiana. Preguntarse por el significado de la llustración es atreverse a dar cuenta de las condiciones de emplazamiento del momento histórico presente. Asimismo, animarse a enmarcar el hoy bajo la mirada filosófica implica, necesariamente, situarse en la vertical del presente y formular la pregunta acerca de ¿qué es nuestro presente? Para Foucault el significado de la pregunta por la llustración radica en el hecho mismo de la posibilidad de su formulación. ¿Qué es la Ilustración? Una nueva forma de plantear, en el orden filosófico, el problema del presente.

\footnotetext{
${ }^{1}$ Foucault, M. El gobierno de sí y de los otros, Bs. As., FCE, 2009, p. 39.
} 
Nueva Otinexaxio Revista Digital de OFilosofla ISSN 1850-3578 2013 - Vol. 8 - Número VIII - Resistencia, Chaco, Argentina. Pp. 136 - 147

Si para el filósofo francés la pregunta por la llustración expresa el ethos filosófico de la modernidad, el animarse a razonar sobre las condiciones del presente, para Kant la pregunta por la llustración se aloja en el hueco abierto por su filosofía de la historia. En efecto, la pregunta por el presente histórico es contemporánea de otro texto kantiano: Idea de una historia universal desde el punto de vista cosmopolita (1784). En este escrito Kant sostiene, de manera general, que: a) las acciones humanas (tomadas en conjunto) están determinadas por leyes de la Naturaleza, de manera que, pensando que son libres, los hombres siguen las intenciones de la Naturaleza; b) una mirada sobre la historia nos puede mostrar que existe una cierta regularidad en las acciones de los hombres y c) que dicha regularidad se caracteriza por un progresivo desarrollo de lo que Kant llama disposiciones originarias del género humano. ${ }^{2} \mathrm{Y}$ para especificar dichas generalidades, Kant esboza una "Idea" de la historia universal a partir de nueve principios. Los mismos establecen que: a) la humanidad ha progresado, en el orden moral, a lo largo de los tiempos (3r. Principio); ${ }^{3}$ b) la causa del progreso moral de la humanidad es el conflicto de egoísmos, la guerra, el antagonismo, la rivalidad, el afán de poder, etc. Los mismos aparecen como el medio del cual la Naturaleza se vale para el desarrollo moral del conjunto de los hombres (4‥ Principio); ${ }^{4} \mathrm{c}$ ) el sentido del progreso moral de la humanidad está puesto en su finalidad: una constitución política perfecta (8ㅇ. Principio)..$^{5}$

En el cuadro de esta concepción de la historia es que Kant se pregunta, en el mismo año, sobre el significado de la llustración, es decir, por el sentido de su presente histórico. $Y$ es a partir del ensayo de respuesta que ofrece a dicha cuestión que la Ilustración aparece bajo la forma de un proceso, un proceso a través del cual la humanidad pasa de un estado de minoría de edad a un estado de mayoría de edad. No estamos en una época ilustrada, pero sí en una época de Ilustración, dice Kant hacia el final del texto, dando cuenta de las ventajas prodigadas por el gobierno de Federico el Grande a la publicidad de las ideas doctas.

\footnotetext{
${ }^{2}$ Kant, I. "Idea de una historia universal desde el punto de vista cosmopolita", en Kant, I. Filosofía de la Historia, La Plata, Terramar, 2004, p. 17.

3 Ibid., p. 20.

${ }^{4}$ Ibid., p. 21.

${ }^{5}$ Ibid., p. 28.
} 
Q Nueva Etinexaxio Revista Digital de OFilosofia ISSN 1850-3578 2013 - Vol. 8 - Número VIII - Resistencia, Chaco, Argentina. Pp. 136 - 147

Pero si el texto sobre la llustración aparece como una constatación del devenir progresivo de la historia planteado también en el escrito sobre la historia universal, la pregunta sobre las garantías de tal conjunto de aseveraciones en el plano teórico se plasma como correlato de cada afirmación vertida. Teniendo presente las limitaciones establecidas por el propio Kant al despliegue de la razón teórica, ¿es posible un conocimiento del devenir que no viole los postulados de la Crítica de la Razón Pura? Pese al esfuerzo de Kant por acercarse a alguna forma de respuesta positiva, la conclusión parece situarse del lado de la negativa (al menos en este texto del año 1784). En Idea de una historia universal... señala:

Pero ahora se trata de saber si la experiencia descubre algo de la marcha de semejante intención de la Naturaleza. Digo que muy poco; pues, antes de cerrarse, esta órbita parece exigir tanto tiempo que sólo podremos, basándonos sobre la pequeña parte que la humanidad ha recorrido en ese sentido, determinar la forma de la trayectoria y la relación de las partes con el todo, aunque con tan poca seguridad como si quisiéramos establecer el curso que el sol y todo el cortejo de sus satélites siguen en el gran sistema de las estrellas fijas, a partir de las observaciones del cielo hasta ahora realizadas. ${ }^{6}$

A través de una analogía con los estudios astronómicos Kant trata de mostrar la imposibilidad de tener algún tipo de certeza acerca de la finalidad hacia la que la Naturaleza arrastra a los hombres. Tanto el curso del Sol y de sus planetas, sobre el fondo de las estrellas fijas, así como el derrotero de la Naturaleza presuponen, para revelarse, temporalidades que exceden ampliamente la vida de un hombre e incluso la de la especie humana. Podríamos, incluso, llegar a establecer el sentido de la marcha de la historia en una pequeña porción del devenir de la humanidad, sin embargo, esto no sería garantía suficiente para, a partir del mismo, conocer la intención de la totalidad del Plan de la Naturaleza.

Pero si no podemos esgrimir un conocimiento teórico acerca del curso de la historia, entonces ¿qué significa la pregunta de Kant por la llustración? ¿Qué tipo de pregunta

\footnotetext{
${ }^{6}$ Ibid., p. 28.
} 
Q Nueva Etinexaxio Revista Digital de OFilosofia ISSN 1850-3578 2013 - Vol. 8 - Número VIII - Resistencia, Chaco, Argentina. Pp. 136 - 147

es aquella que inquiere por el sentido de la época desde un lugar que no está emplazado en los órdenes del conocimiento teórico? ¿En dónde se ha instalado la pregunta si no es en el plano del saber? Frente a la dificultad de conocer y predecir el curso de la historia, Kant sostiene, en Idea de una historia universal..., que:

\begin{abstract}
...aunque seamos demasiado miopes como para penetrar en el mecanismo secreto de esa organización [la Naturaleza], tal idea podría servirnos, sin embargo, de hilo conductor para exponer, por lo menos en sus lineamientos generales y como sistema, lo que de otro modo no sería más que un agregado sin plan de las acciones humanas. ${ }^{7}$
\end{abstract}

Pensar que la humanidad se halla en constante progreso hacia una finalidad prometedora es una "idea" práctica que, de hecho, abre -y Kant lo menciona a continuación de lo citado anteriormente- “...una consoladora perspectiva para el futuro". ${ }^{8}$ No solo la esperanza de un porvenir mejor, en el orden de la legalidad, puede construirse a la luz de la idea de un Plan de la Naturaleza, sino, al mismo tiempo, la necesidad de trabajar por la concreción del mismo. Es en la disposición de objetos en este cuadro que el escrito kantiano sobre la llustración se instala como un ejercicio del uso público de la razón, ejercicio que es la condición misma de la salida de la minoría de edad. En esta perspectiva, el texto de Kant funciona "estratégicamente", pudiéndose enmarcar en lo que Foucault, en la conferencia de 1978, dice sobre dicho escrito:

En consecuencia, esta definición de la Aufklärung no va a ser simplemente una suerte de definición histórica y especulativa; habrá algo, en ella que, sin duda, resultaría ridículo llamar prédica; es más bien, en todo caso, un llamado al coraje que Kant lanza en esa descripción de la Aufklärung. ${ }^{9}$

\footnotetext{
${ }^{7}$ Ibid., p. 30.

8 Ibid., p. 31.

9 Foucault, M. “Crítica y Aufklärung [“Qu'est-ce que la Critique?”]", en: Revista de Filosofía, trad. Jorge Dávila, Venezuela, Universidad de Los Andes, 1995, $\mathrm{N}^{\circ} \quad 8 . \quad$ Disponible en: http://www.saber.ula.ve/bitstream/123456789/15896/1/davila-critica-aufklarung.pdf, p. 4. Consultado el 15-07-2013. Texto Original en Bulletin de la Société Française de Philosophie, 84ㅇ année, № 2, AvrilJuin 1990, pp. 35-63.
} 
Q Nueva Etinexaxio Revista Digital de OFilosofia ISSN 1850-3578 2013 - Vol. 8 - Número VIII - Resistencia, Chaco, Argentina. Pp. 136 - 147

Sin embargo, el llamado a armarse de coraje, acentuado por Foucault, no parece satisfacer del todo a Kant en cuanto a ser una condición suficiente para lograr el fin de la Naturaleza. En El conflicto de las facultades, un texto de 1798 y, por ende, bien posterior a Idea de una historia universal, Kant se vuelve a preguntar si el género humano se halla en constante progreso hacia lo mejor. Para responderse, establece algunas precisiones del tipo: a) es en el orden moral de la humanidad que el progreso se hace visible; b) el saber acerca de dicho progreso no es algo que nos venga dado por la experiencia, es decir, se pueden disponer los acontecimientos de manera tal de esperar que en el futuro las acciones tomen una determinada senda, pero al final de cuentas nada nos asegura que dicho recorrido se cumpla. Dice Kant: "La cuestión del progreso no se puede resolver directamente por la experiencia... Hemos de enfrentarnos con seres que actúan libremente. Por cierto, se les puede dictar de antemano lo que deben hacer, pero no se puede predecir lo que harán..." ${ }^{10}$

Sin embargo, el futuro no puede quedar librado a la contingencia de los vaivenes de la historia. Si bien la predictibilidad de los acontecimientos venideros -a la manera en que la entienden las ciencias naturales- sea algo que no pueda aplicarse de manera rígida al devenir de la historia, entre el punto 5 y 6 de la Reiteración de la pregunta... Kant establece que las narraciones de tipo proféticas, como la que él mismo está desarrollando acerca del progreso moral de la humanidad, deben poder anclarse en algún tipo de experiencia: "En el género humano tiene que acaecer alguna experiencia que, como acontecimiento, se refiera a cierta aptitud y facultad de ser causa de su progreso a lo mejor...". ${ }^{11}$

En el mismo texto, un poco más adelante, da forma al asunto afirmando que:

...se puede predecir un acontecimiento como efecto de una causa dada cuando suceden las circunstancias que cooperan en ello. Que las últimas [circunstancias]

\footnotetext{
${ }^{10}$ Kant, I. "Reiteración de la pregunta de si el género humano se halla en constante progreso hacia lo mejor", en Kant, I. Filosofía de la Historia, ob. cit., p. 154.

${ }^{11}$ Ibid., p. 155.
} 
Q Nueva Etinexaxio Revista Digital de OFilosofia ISSN 1850-3578 2013 - Vol. 8 - Número VIII - Resistencia, Chaco, Argentina. Pp. 136 - 147

tengan que concurrir alguna vez, es algo que se puede predecir en general, como acontece, en los juegos de azar, con el cálculo de probabilidades; ... ${ }^{12}$

En conclusión, y pese a las dificultades expresadas con respecto a conocer el fin de la marcha de la Naturaleza, que surgían de los pasajes de Idea de una historia universal..., pareciera que, según el Kant de Reiteración de la pregunta..., al menos es posible predecir el acaecer de las circunstancias que alentarían una relación causal bajo la óptica del cálculo de probabilidades. Sin embargo, poco se dice aquí acerca de cuáles podrían ser esas circunstancias, predecibles desde un régimen probabilístico, y tan determinantes para enlazar una causa basada en aptitudes y facultades del género humano con un acontecimiento determinado.

Ahora bien, si es posible predecir, de manera probabilística, el concurso de las circunstancias que favorecerán que un acontecimiento se enlace, en tanto efecto, a una causa dada, entonces se puede predecir, también dentro de un margen de probabilidades, que tal acontecimiento se efectuará. Aquí el problema de poder aseverar algo con certeza acerca del curso de los acontecimientos históricos parece tomar aliento. Frente a la imposibilidad de establecer el carácter necesario del curso del devenir de la historia, presente en los textos anteriores, ahora se hace posible apelar al cálculo de probabilidades para que nos brinde un margen aproximado de referencia acerca de la posible concreción de ciertos acontecimientos. El único problema que Kant conserva con respecto a este asunto es que si bien las probabilidades dicen que algo podría pasar, de acuerdo con ciertas variables, no establecen cuándo. Y allí, en ese punto, Kant encuentra un límite para el estatuto del conocimiento teórico de lo que está sosteniendo en estos textos acerca del sentido de la historia: “...pero no puede predeterminarse si eso pasará en mi vida y si tendré la experiencia que confirme tal previsión" ${ }^{13}$ La finitud de la vida se convierte en una variable negativa ante la necesidad de una confirmación empírica del conocimiento planteado en el orden probabilístico.

\footnotetext{
12 Ibid., p. 156.

${ }^{13}$ Ibid., p. 156.
} 
Q Nueva Etinexaxio Revista Digital de OFilosofia ISSN 1850-3578 2013 - Vol. 8 - Número VIII - Resistencia, Chaco, Argentina. Pp. 136 - 147

Para que el progreso de la historia pueda ser asumido como conocimiento teórico la profecía y la realización de la misma deben estar ligadas por la presencia del mismo sujeto de experiencia. Y es en torno de este problema que Kant establece la necesidad de:

\footnotetext{
...buscar un acontecimiento que indique, de modo indeterminado con relación al tiempo, la existencia de una causa semejante y también el acto de su causalidad en el género humano, de modo que permita inferir, como inevitable consecuencia, el progreso hacia lo mejor. ${ }^{14}$
}

Ahora bien, ¿̇qué acontecimiento puede indicar, "de modo indeterminado con relación al tiempo", puesto que será difícil olvidarlo, la existencia de una causa vinculada al género humano que dé cuenta del progreso hacia lo mejor? La Revolución Francesa da una pista con respecto al acontecimiento que Kant está buscando. La adhesión que la misma genera a través de las letras en buena parte de Europa se vuelve un acontecimiento-signo, puesto que: a) es un acontecimiento que remite -en tanto efecto- a una causa vinculada a la humanidad y, en tanto tal, b) es indicativo -signo- de una marcha progresiva de la historia. ${ }^{15}$ Pero, ¿cuál es la causa que, bajo ciertas circunstancias, puede producir un acontecimiento de la magnitud del que se desarrolla alrededor de la Revolución Francesa? La disposición moral del género humano, ciertamente. La misma se evidencia no sólo en los hombres que llevaron adelante la Revolución, sino en todos aquellos que ven con simpatía el acaecimiento de la misma, que hacen pública su toma de posición a favor de los intereses revolucionarios y que, aunque jamás levantarían una barricada ni integrarían las filas de una muchedumbre encendida, expresan la absoluta conveniencia de estos acontecimientos. Esa disposición moral del género humano que obra como causa y que posibilita, a través de la circunstancia de la Revolución Francesa, la adhesión manifiesta a ella, se condensa, para Kant, en un deseo que no puede dejar de dirigirse a un Estado de Derecho y a una Constitución Republicana. En poco tiempo los motivos de la

\footnotetext{
${ }^{14}$ Ibid., p. 156.

${ }^{15}$ Ibid., p. 156.
} 
eNueva Etinexaxio Revista Digital de OFilosofla ISSN 1850-3578 2013 - Vol. 8 - Número VIII - Resistencia, Chaco, Argentina. Pp. 136 - 147

Revolución Francesa encuentran un eco extendido de aprobación, expresado a través del uso público de la razón, en distintos Estados. Pero Kant cree que los deseos republicanos que desencadenaron la Revolución, y los de aquellos espíritus que se han volcado a su calurosa aprobación, no dejarán de crecer y extenderse, puesto que “...cuando acaece un fenómeno como ése en la historia humana, ya no se olvida jamás". ${ }^{16}$ La Revolución se convierte en la circunstancia que permite enlazar las disposiciones racionales y morales de la humanidad con un acontecimiento (el despliegue del espíritu revolucionario-republicano) que se transforma en signo de la inexorable marcha de la Naturaleza hacia una Constitución Republicana y una Confederación de Naciones. En tanto circunstancia, la revolución es un fenómeno que se puede preveer según el cálculo de probabilidades, de manera semejante a la manera en que el mismo determina las frecuencias en que determinados valores de un dado pueden salir tras una cierta cantidad de lanzamientos. Las matemáticas probabilísticas de Jakob Bernoulli, pero especialmente el aporte de Laplace sobre el uso de las probabilidades para conjurar la ignorancia irremediable acerca de la estructura causal y determinista de la Naturaleza, introducen una herramienta teórica que permite establecer patrones de regularidad en acontecimientos que muestran un alto grado de indeterminación. La relación entre la aplicación del cálculo de probabilidades a la predicción de acontecimientos astronómicos y naturales y su posible entrecruzamiento con el problema filosófico de una organización causal del devenir es algo que convendría profundizar más. Sobre todo, si tenemos presente que un problema común a Kant y Laplace es el de cómo salvar la distancia que existe entre la finitud de la condición humana y la organización racional del todo de la Naturaleza. Las probabilidades permitirán a Laplace encontrar pistas en aquellos lugares en los que la predicción absoluta es imposible. Para Kant, la posibilidad de la constitución de acontecimientos-signo se volverá el baremo que permitirá predecir el derrotero de la Historia hacia un fin. De aquí que Kant concluya el texto Reiteración de la pregunta... sosteniendo, en forma confiada:

\footnotetext{
${ }^{16}$ Ibid., p. 159.
} 
Q Nueva Etinexaxio Revista Digital de OFilosofia ISSN 1850-3578 2013 - Vol. 8 - Número VIII - Resistencia, Chaco, Argentina. Pp. 136 - 147

Ahora bien, aún sin espíritu profético, y de acuerdo con los aspectos y signos precursores de nuestros días, afirmo que puedo predecir que el género humano logrará esa meta y también que sus progresos hacia lo mejor ya no retrocederán completamente. ${ }^{.17}$

Esta afirmación es cualitativamente diferente de aquellas que planteaba en Idea de una historia universal... No es ni un hilo conductor para sistematizar el devenir ni una estrategia práctico-política que nos permita seguir esperanzados en la consolidación de una sociedad organizada de acuerdo con una forma republicana. Al contrario, sostiene Kant que "el sostén de esta tesis no se halla en alguna proposición bienintencionada y prácticamente recomendable, sino que tiene vigencia, a pesar de los incrédulos, en la más rigurosa teoría...". ${ }^{18}$ Ahora bien, ¿en dónde reside la garantía de esta tesis sobre el devenir teleológico de la historia? Kant responde, hacia el final del texto en cuestión, retomando los parámetros $y$, por ende, el estatuto del conocimiento teórico expuesto en la primera Crítica: "sólo contamos con datos empíricos (experiencias) para fundamentar esa profecía, a saber, sobre la causa física del acontecer de nuestras acciones, por lo cual también son fenómenos" ${ }^{19}$

Ahora bien, en el marco del proyecto foucaultiano de una ontología histórica de nosotros mismos, podemos preguntarnos acerca de la operación de lectura que Foucault realiza sobre el texto kantiano de la llustración. Si se recorren los escritos sobre la Aufklärung que el filósofo francés elabora entre 1983 y 1984 es posible advertir que la operatoria fundamental consiste en recortar el ensayo de 1784 del fondo de problemas relativos a la filosofía de la historia, que caracterizan buena parte de la producción de Kant hasta 1798. La tesis de una historia que deviene de acuerdo con un fin no permite articular una ontología del presente cuyo objetivo sea dar cuenta de los límites históricos que nos constituyen a efectos de poder desplegar experiencias-experimentos para franquearlos. El ejercicio del franqueamiento posible de nuestra actualidad queda bloqueado desde el momento en que una filosofía de la historia establece la dirección que el curso de los acontecimientos debe seguir. En

\footnotetext{
17 Ibid., p. 159.

${ }^{18}$ Ibid., p. 160.

${ }^{19}$ Ibid., p. 163.
} 
eNueva Etinexaxio Revista Digital de OFilosofla ISSN 1850-3578 2013 - Vol. 8 - Número VIII - Resistencia, Chaco, Argentina. Pp. 136 - 147

palabras de Kant: "cuando digo de la Naturaleza que quiere que ocurra esto o lo otro, no quiero decir que nos impone un deber de hacerlo (pues ello sólo puede hacerlo la razón práctica, que es libre de toda coacción). Lo que quiero decir es que lo hace por sí misma, lo queramos o no...." ${ }^{20} \mathrm{Y}$ citando la máxima del estoicismo agrega: "fata volentem ducunt, volentem trahunt" (el destino guía al que lo acepta y arrastra al que se resiste).

La gran apuesta foucaultiana alrededor del texto kantiano consiste en encolumnar a Kant en la hilera de aquella tradición que giraría alrededor de un nuevo modo de problematización del presente, al precio, claro está, de desbaratar el pensamiento acerca de la necesidad en el curso de la historia. La pregunta que queda flotando en el aire es ¿acaso no hubiera implicado un rodeo menor recuperar de Nietzsche la idea de un diagnóstico del presente y de lo que somos, a efectos de una transformación posible? ¿Por qué la opción foucaultiana por transitar el camino lleno de escollos, a través de Kant, y no el recorrido más ameno a lo largo de Nietzsche?

\footnotetext{
${ }^{20}$ Kant, I. Hacia la paz perpetua. Un proyecto filosófico, Bernal, Universidad Nacional de Quilmes, Bs. As., Prometeo Libros, 2007, p. 74.
} 